\title{
Content Based Image Retrieval Using K-Means Algorithm
}

\author{
Shubhi Misra ${ }^{1}$, Atul Kumar Verma ${ }^{2}$ \\ ${ }^{1}$ Department of Computer Science and Engineering, \\ Shri Ramswaroop Memorial College of Engineering and Management, Lucknow, Uttar Pradesh, India. \\ ${ }^{2}$ Faculty, Department of Computer Science and Engineering, \\ Shri Ramswaroop Memorial College of Engineering and Management, Lucknow, Uttar Pradesh, India.
}

\begin{abstract}
The importance of information sharing through images is widespread among individuals. The community/social networking facilitates the users to upload, download and share various images. It is a complex task to segregate an image from image rich information network. This paper's main motive is to evaluate the performance of CBIR system and to find relevant image from annotated images that match query and assign right name label. K-means clustering algorithm is proposed as it improves the scalability. It includes combine theories of CBIR and analysis of features of CBIR system.
\end{abstract}

Keywords: CBIR; K-means; Feature Extraction; Image annotation; Image indexing.

\section{INTRODUCTION}

In today's era, images play a wide role in major part of life. Due to technological advancement in digital camera, speedy network and social networking sites, the images have been the foremost information holder and sharer, be it any important message or the photographs of individuals.

The social sites accompany the images with all the links, annotations, comments and tags which forms an image rich information network. Information retrieval in image rich source is very challenging task [1].

There are two methods which can be carried on. Firstly, the query image can be given the correct annotation after matching it with the dataset. Secondly, the query image can retrieve a number of similar images after matching it with dataset. Annotation means the process of naming a picture from the large dataset of images. Annotation involves semantically labelling the image with correct label in a huge database as this facilitates the information retrieval from the image rich information network and provides large potential applications. The need of annotation arises due to increasing growth of image based information sharing specially in social networking [1].

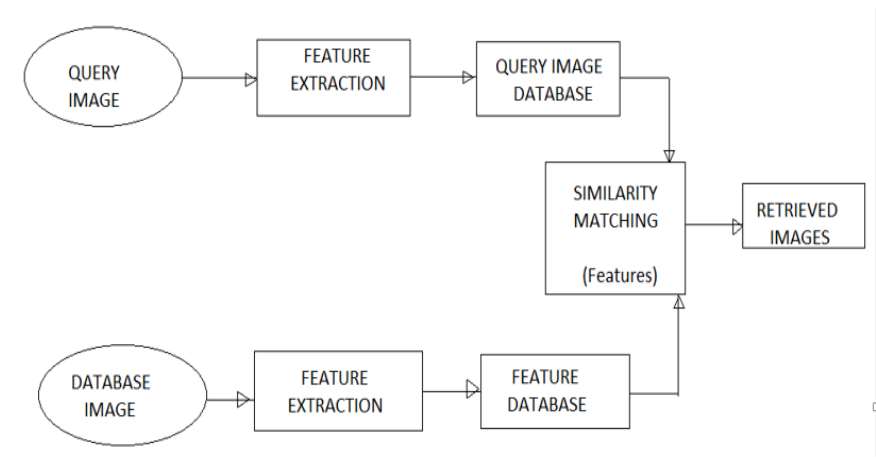

Figure 1: Block diagram of CBIR system

\section{LITERATURE REVIEW}

Snehal Yadav, Sneha Varne, Neha Jadhav, Shamal Powar and Priyanka Patil [1] have proposed the S-CBIR algorithm technique. Since, different re-ranking techniques have limitations such as computationally expensive and not very practical, the S-CBIR technique is used. It includes the three main steps. Firstly, we load all images from dataset and their tag id's. The indexing of common tag images is done and K-means algorithm is computed. Then the images are extracted and stored the image features in dataset. Secondly, we get an input tag entry which we call a keyword. If the keyword is present in dataset then we fetch the tag id's for that tag. We find image id's and fetch K-means for all the image nodes to get score and then display images. Lastly, we select an image as input query and perform basic feature extraction, color extraction, texture extraction, sort them and display images. The accuracy in this approach is much improved. This scheme provides semantically and visually similar images as output.

Suchita Barkund and Dr. Sulochana Sonkamble [2] presents a Search-Based Face Annotation (SBFA) technique which uses content-based image retrieval (CBIR) technique in the mining of facial images of human beings. To assign correct name labels to a given query facial image which is motivated by SBFA. The efficient image decomposition, feature extraction and image matching mechanisms performed by CBIR and annotation of user well performed. The additional work improves the efficiency of the system in which identify the users by performing email verification and last login.

Rinku Avinash Saoji and Prof. M.K. Kodmelwar [3] presents an image retrieval system in which features are generated from 
ODBTC and indexing of image in database is done. The ODBTC compresses image into a set of color quantizers and bitmap image. The color co-occurrence feature and bit pattern feature are generated from color quantizer and bitmap image respectively. The color co-occurrence feature and bit pattern feature extraction is applied on query image and the images from image database are taken and their features are extracted and similarity computation is done.

Poonam Bhatia Anand and Neetu Bhatia [4] have discussed many features used in CBIR systems. It can be drawn from the analysis that the wide variety of features are used by the QBIC CBIR systems. QBIC uses both level of features as there are two types of features used in CBIR system i.e. high level and low level features.

Devrat Arya and Jaimala Jha [5] describes image enhancement. Of both the query image and collected images, image pyramid is made (designed) on data structure to support effective scaled convolution by decrease image representation. After this the color quantization and color space is performed. In quantization the various distinct colors of an image is decreased and in HSV, the perception value is achieved. Since, HSV is general method to extract color feature of an image, therefore, GCV is used to extract color feature of an image pyramid. DWT is implemented to decrease computation time and resources need. Since, in $\mathrm{GCV}$, the color information can be lost directly so DGCV is proposed to extract features of filtered images. After this, the similarity matching is done using Euclidean distances and the correct image/images are retrieved.

Kratika Sharma and R.K Gupta [6] use different types of distances to calculate similarity between two images. This approach focuses only on retrieval of image files. A set of images is stored in database and its features are extracted i.e contrast enhancement and stretching of resized image is done. Similar thing is done with the query image. Adaptive K-means algorithm is used to obtain connected components and to convert RGB color format to HSI. The mean, standard deviation, smoothness of image, degree of asymmetry is found. The similarity between images is obtained through using different methods. Classification of images are done by combining low level features.

Nidhi Tripathi, Pankaj Sharma and Manish Gupta [7] performs pre-processing, feature extraction and classification. The image is stretched and resized with adjustment of image contrast. The $\mathrm{RGV}$ color format is changed to HSV color space. Various techniques relevant to different operations are used.

Aman Saini and Sonit Singh [8] have done system evaluation on the basis of precision, recall, NMRR and retrieval time. The features of an image are extracted by using three different techniques and their combination. The retrieval results based upon texture by using GLCM are quite good but this approach takes maximum time in providing retrieval results at the output due to the complexity involved in calculation of feature vectors.

\section{PROPOSED METHODOLOGY}

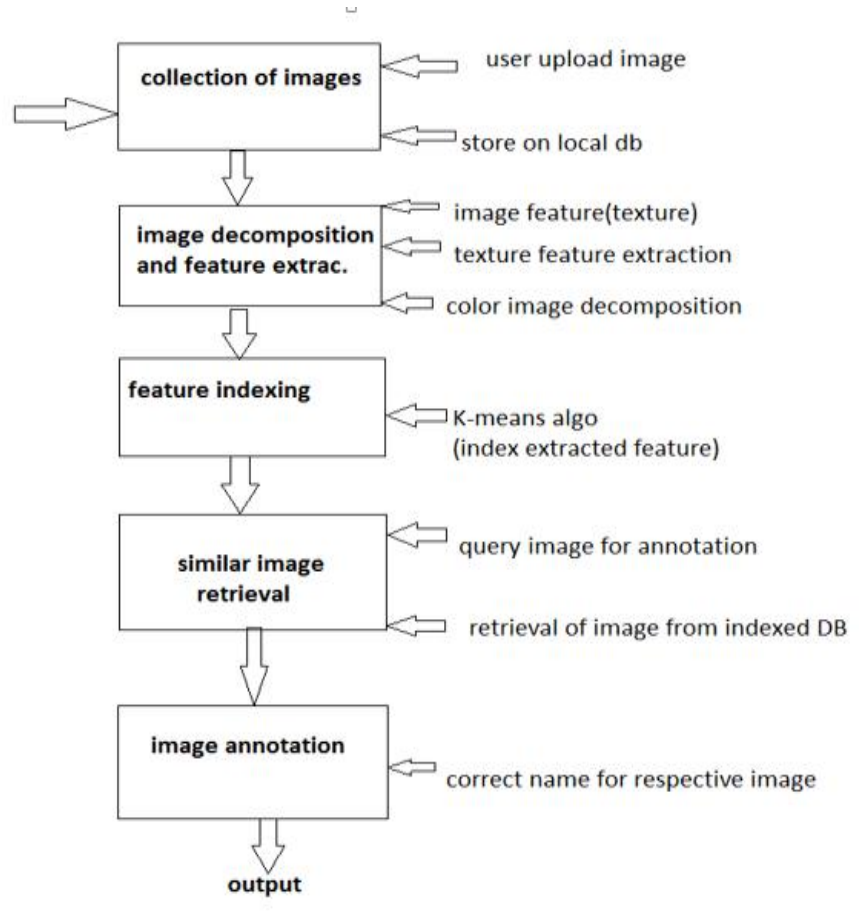

Figure 2: Data Flow Diagram

\section{A. K-means Clustering Algorithm:}

Clustering is the process of grouping of the objects on the basis of a close association or shared characteristics. The objects can be physical or abstract entities, and the characteristics can be attribute values, relation among the objects or combination of both. In clustering, the similarity patterns are found among a group of objects.

K-means clustering is an unsupervised learning method of cluster analysis. It is explained as:

1. The basic idea of this is that clusters with same target category are identified and predictions for new data items are made by assuming that they are of the same type as nearest cluster center.

2. Suppose we have $m$ feature vectors $\left(a_{1}, a_{2} \ldots a_{m}\right)$ all belonging to same class " $\mathrm{C}$ " and we know that they belong to " $\mathrm{z}$ " clusters such that $\mathrm{z}<\mathrm{m}$.

3. If clusters are well separated, we can use a minimum distance classifier to separate them.

4. We first initialize the means of " $z$ " clusters which are $\mu_{1}$, $\mu_{2} \ldots \mu_{\mathrm{z}}$. One of the way to do this is to assign random numbers to them.

5. We then determine the membership of "a" by taking the $\left|a-\mu_{i}\right| \mid$, where $(i=0,1 \ldots z)$.

6. The minimum distance determines a's membership in a respective cluster. This is done for all " $\mathrm{m}$ " feature vectors. 


\section{B. The Algorithm:}

The algorithm is composed of the following steps:

1. Place " $z$ " points into the space represented by the objects that are being clustered. These points represent initial group centroids.

2. Assign each object to the group that has the closest centroid.

3. When all objects have been assigned, recalculate the positions of the " $z$ " centroids.

4. Repeat step 2 and step 3 until the centroids no longer move.

\section{EXPECTED RESULT}

The performance evaluation is shown in fig. 3 .

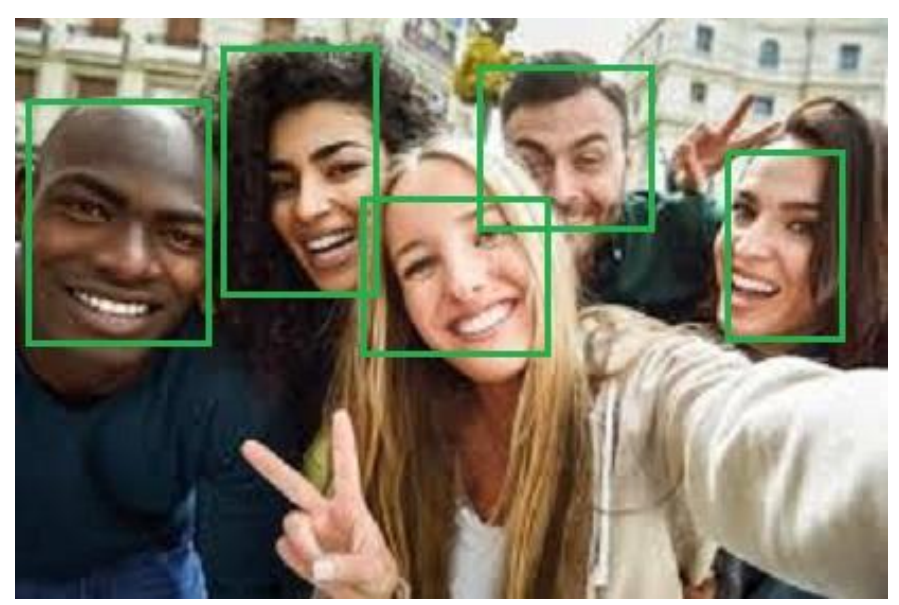

Figure 3: Recognition of facial images

The images are recognized and will be annotated as the possibility of accuracy matching and gathering of information from accurate source which may be any socially active domain. The accuracy of proposed system is better as compared with existing system.

\section{SYSTEM ANALYSIS}

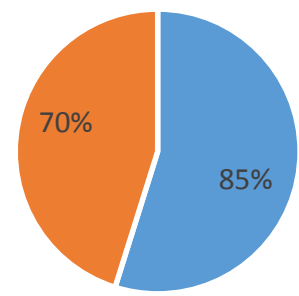

- PROPOSED SYSTEM - EXISTING SYSTEM

Figure 4: Accuracy comparison of proposed system

\section{CONCLUSION}

There are many features of an image used in CBIR system but the techniques to attain perfection are under research. An image retrieval system returns a set of images from a collection of images in a database. In CBIR, visual characteristics such as shape, color and texture are the descriptors to characterize images. The use of K-means clustering algorithm improves the scalability. So, the system helps to develop an environment where relevant images can be segregated and also correct name given to an image input.

\section{FUTURE SCOPE}

The accuracy of the system may be improved by combining it with some other feature extraction techniques, machine learning or by using user feedback. Some distance measurement techniques may be used to speed up the processing and retrieval of images. The combined techniques are expected to provide a better result than a single precision technique.

\section{REFERENCE}

[1] Snehal Yadav, Sneha Varne, Neha Jadhav, Shamal Powar and Priyanka Patil "Improved Accuracy of Image Retrieval by using K-CBIR”, IRJET, Volume:03 Issue:04, April-2016, pp-2343-2345.

[2] Suchita Barkund and Dr. Sulochana Sonkamble "Search-Based Face Annotation with CBIR and Clustering-based Algorithm", IRJET, Volume:03 Issue:06, June-2016, pp-2204-2208.

[3] Rinku Avinash Saoji and Prof. M.K. Kodmelwar "Survey on CBIR using Halftoning BTC", IJARCSM, Volume:04 Issue:1, June-2016, pp-42-45.

[4] Poonam Bhatia Anand and Neetu Bhatia "Feature Analysis in CBIR Systems", IJCATR, Volume:05 Issue:06, pp-358-363.

[5] Devrat Arya and Jaimala Jha "Global and Local Descriptor for CBIR and image enhancement using multi feature fusion methd", Arya et. al., Volume:04 Issue:06, June-2016, pp-170-182.

[6] Kratika Sharma and R.K Gupta "A Novel CBIR System Using Adaptive K-Means Clustering and Multi Class SVM Classification", IJASRT, Volume:02 Issue:06, June-2016, pp-389-395.

[7] Nidhi Tripathi, Pankaj Sharma and Manish Gupta "A New Technique for CBIR with Contrast Enhancement using Multi-Feature and Multi Class SVM Classification", IEEE, 2016.

Aman Saini and Sonit Singh "Evaluation of CBIR System by Using Low Level Features of an Image", IJSETR, Volume:05, Issue:06, June-2016,pp-19511954. 